Cinémas

Revue d'études cinématographiques

Journal of Film Studies

\title{
Deleuze et la question de la narration
}

\section{Marion Froger}

Volume 10, numéro 1, automne 1999

Cinélekta 3

URI : https://id.erudit.org/iderudit/024807ar

DOI : https://doi.org/10.7202/024807ar

Aller au sommaire du numéro

\section{Éditeur(s)}

Cinémas

\section{ISSN}

1181-6945 (imprimé)

1705-6500 (numérique)

Découvrir la revue

Citer cet article

Froger, M. (1999). Deleuze et la question de la narration. Cinémas, 10(1),

131-155. https://doi.org/10.7202/024807ar

\section{Résumé de l'article}

L'objet de cet article est une mise au point sur les principaux concepts que Deleuze utilise pour explorer le champ ordinairement investi par la narratologie filmique. On entend ici dresser la carte d'un parcours singulier où Gilles Deleuze commence par se débarrasser du bagage linguistique légué par Metz et la critique littéraire, pour revenir aux sources du récit filmique, à ces sortes de mouvements qui le font naître et qui lui déploient un monde où, incidemment, il lui arrive de se suspendre, de se détourner, de se perdre. Il sera question de la différence entre " histoire " et " devenir », de la distinction entre deux sortes de récits, récit et narration falsifiants, récit et narration véridiques, et de l'indiscernabilité comme principe d'une image-cristal née du cinéma moderne, qui change les modalités de la réception, ainsi que les outils et méthode, de l'analyse. 


\section{Deleuze et la question de la narration}

\section{Marion Froger}

\section{RÉSUMÉ}

L'objet de cet arricle est une mise au point sur les principaux concepts que Deleuze utilise pour explorer le champ ordinairement investi par la narratologie filmique. On entend ici dresser la carte d'un parcours singulier où Gilles Deleuze commence par se débarrasser du bagage linguistique légué par Metz et la critique littéraire, pour revenir aux sources du récit filmique, à ces sortes de mouvements qui le font naître et qui lui déploient un monde où, incidemment, il lui arrive de se suspendre, de se détourner, de se perdre. Il sera question de la différence entre "histoire" et "devenir ", de la distinction entre deux sortes de récits, récit et narration falsifiants, récit et narration véridiques, et de l'indiscernabilité comme principe d'une imagecristal née du cinéma moderne, qui change les modalités de la réception, ainsi que les outils et méthode, de l'analyse.

\section{ABSTRACT}

This article has as its object a clarification of the main concepts used by Deleuze to explore the field which has usually been the domain of film narratology. What we aim to do here is to map out a singular itinerary, from the moment when Deleuze steers away from the linguistics inherited from Metz and literary criticism in order to return to the sources of film narrative: the sort of movements which create film narrative and give it a world where it may sometimes, incidentally, suspend itself, make detours, or lose itself. At stake is the difference between "history" and "evolution." This is 
also the distinction between two types of narratives and narration, the falsifying and the truthful, as well as indiscernibility as the principle of an image-crystal born of modern cinema, which changes both the modalities of reception and the tools and method of analysis.

S'il y a des philosophes à qui l'on fait porter tous les chapeaux, Gilles Deleuze serait plutôt l'un de ceux à qui l'on retire toutes les casquettes, surtout dans le cénacle des études cinématographiques, où l'on prit l'habitude de limiter la portée théorique de ses réflexions sur le cinéma, sous prétexte que sa "philosophie", "son esthétique" occuperaient en leur déploiement le champ justement délaissé sciemment, et pour cause de rationalité scientifique, par les théoriciens. Pourtant, bon nombre des concepts deleuziens (sa nomenclature des images, ses figures du montage, etc.) se retrouvent çà et là, de manière dispersée, dans les écrits théoriques consacrés au $7^{\mathfrak{e}}$ art. Mais ces apparitions conceptuelles sont rarement systématisées, et dans certains champs bien définis des études cinématographiques, comme dans celui de la narratologie filmique que touche notre étude, il est à peine fait allusion à L'Image-temps et L'Imagemouvement. Ainsi, on a rarement pris la peine d'interroger les propositions deleuziennes concernant le récit et la narration, c'est-à-dire la raison d'une telle divergence de vue, de positionnement et de perspective avec les développements de la narratologie filmique. Il faut dire que Gilles Deleuze n'y va pas de sa contribution à la résolution des questions que soulevèrent les premières propositions de Christian Metz. On ne retrouve chez ce philosophe aucune des interrogations ' que Michel Marie dénombre dans la somme que François Jost et André Gaudreault consacrent au récit et à la narration au cinéma. Est-ce à dire que la position deleuzienne serait complètement "hors champ", et ne répondrait qu'à d'obscures motivations philosophiques dont les narratologues n'ont finalement que faire?

Certes, Gilles Deleuze n'est pas un narratologue. L'analyse qu'il conduit dans L'Image-mouvement et L'Image-temps ne prend pas directement pour objet le récit et la narration. Elle les rejoint plutôt, sans avoir adopté ni les présupposés ni les 
distinctions qui fondent et jalonnent les approches narratologiques traditionnelles. Les pages qui vont suivre seront donc motivées par la question suivante: quel regard oblique Gilles Deleuze jette-t-il sur la narration et le récit, et quelles sont les pistes théoriques qu'il s'est plu à dégager? Il s'agit là moins d'un commentaire que d'une mise en perspective de quelques propositions, afin de leur faire prendre part au débat des narratologies constituées, et comme pour participer, d'une touche deleuzienne, au mouvement actuel de renouvellement théorique de ce champ d'étude. Nous essayerons de proposer une synthèse de cette question narratologique, au risque quelquefois de ne pouvoir que répéter, en simplifiant, le propos du philosophe. Mais c'est un risque bien ordinaire pour ce genre d'entreprise, et que nous assumons volontiers, puisque si Deleuze génère énormément de réflexion chez ses élèves, lecteurs et épigones, le corpus critique qui lui est consacré est encore pauvre en simples tentatives d'explication de texte, au sens scolaire du terme. C'est à l'extension de ce corpus introductif que l'on aimerait ici contribuer.

Pour débuter cette étude sans reprendre un travail de synthèse fort bien fait par ailleurs ${ }^{2}$, nous nous attarderons sur le point suivant: la narratologie classique, qui a nourri les études cinématographiques, développe, comme on le sait, deux approches. La première s'occupe des contenus des récits indépendamment du médium, la deuxième s'attache aux formes d'expression spécifique au médium utilisé. Or, pour ce qui concerne cette dernière approche, la narratologie filmique a beaucoup puisé chez Christian Metz pour définir le médium cinématographique. Et l'on sait combien ce dernier exploita brillamment les concepts et les voies d'analyse de la linguistique. À la lecture des quelques déclarations ${ }^{3}$ qu'il a pu faire sur son travail, il n'échappe à personne que le propos de Gilles Deleuze est justement d'émanciper complètement les études cinématographiques de la linguistique. Il rejette donc en bloc ce parcours théorique et développe pour sa part une sémiotique propre au cinéma qui va lui permettre de parler de la spécificité d'un récit et d'une narration produits par tel ou tel type d'«agencement d'images» singulier. Ainsi, contrairement à Metz, qui pensait que le cinéma 
avait la "narrativité bien chevillée au corps» (p. 52) - expression qui lui vient justement en considérant le fonctionnement linguistique du cinéma -, Deleuze pense que le cinéma produit du récit, narre à l'occasion, mais n'a aucunement besoin d'un "code " pour signifier, encore moins d'un minimum d'articulation narrative qui fonde, dans le propos metzien, l'identification du plan à un énoncé.

[La linguistique] se contente de fournir des concepts qu'on applique du dehors au cinéma, par exemple, "syntagme». Mais du coup l'image cinématographique est réduite à un énoncé, et l'on met entre parenthèses son caractère constitutif, le mouvement. La narration, au cinéma, c'est comme l'imaginaire: c'est une conséquence très indirecte qui découle du mouvement et du temps, non pas l'inverse. Le cinéma racontera toujours ce que les mouvements et les temps de l'image lui font raconter. Si le mouvement reçoit sà règle d'un schème sensori-moteur, c'est-à-dire présente un personnage qui réagit à une situation, alors il y aura une histoire. Au contraire, si le schème sensori-moteur s'écroule, au profit de mouvements non orientés, désaccordés, ce seront d'autres formes, des devenirs plus que des histoires [...] (Deleuze, 1990, p. 85).

Ici, le temps et le mouvement ne sont pas pensés comme des paramètres de la narration (ce qui permet de mesurer, circonscrire une ligne narrative), mais comme des déterminations qui s'actualisent dans l'image cinématographique, et qui dès lors sont capables de faire naître tel ou tel récit. L'articulation en récit est ce qui vient en dernier, à la fin du processus de signification. Le code supposé qui produit des enchaînements narratifs, descriptifs ou discursifs, selon la nomenclature des articulations qui sous-tendent la "Grande syntagmatique", n'est en rien, pour Deleuze, ce qui donne sens aux images.

Notons aussi tout de suite cette distinction que Gilles Deleuze fait entre "histoire» et "devenir", avant d'y revenir plus longuement dans le cours de cette étude. Il s'agit là d'une distinction que n'a certainement pas faite Gérard Genette, et qui va lui permettre de parler d'une narration et d'un récit où vient s'abolir l'histoire: la narration falsifiante, opposée dans son fonction- 
nement et dans ses implications philosophiques à la narration véridique. L'histoire, ou contenu narratif, n'est en effet jamais analysée en tant que telle dans L'Image-mouvement et L'Imagetemps. Même lorsque Gilles Deleuze sera amené à caractériser le cinéma d'action (et à passer dans le champ de la narratologie thématique), il s'écartera des voies tracées par la poétique d'Aristote, ou par le schéma actantiel de Greimas, afin de centrer son intérêt sur la conception du sujet, sur l'«idée » qui importe plus que le scénario, ainsi que sur "la relation" entre un personnage et son milieu, qui innerve les images et les organise en séquence, série, etc., de montage. L'action n'est alors rien d'autre que le dévoilement, ou bien l'abolition, ou la restauration de cette relation. Gilles Deleuze considère donc la sorte de "signes" et "d'agencement de signes" qui expriment, par l'image et le montage, cette relation, plutôt que les aspects formels du récit en tant qu'énoncé de représentation ${ }^{5}$, et les scansions du cours événementiel de l'histoire.

Le fondement de l'analyse deleuzienne, s'il n'est pas "sémiologique», se veut en revanche purement "sémiotique". S'il refuse de considérer le cinéma comme un langage (avec ses agencements paradigmatiques et syntagmatiques), c'est que pour lui, le plus gros reproche que l'on peut adresser à la linguistique est d'avoir occulté et appauvri la notion de signe, dont pour sa part, il va faire un usage extraordinaire, débordant même toutes les sémiotiques connues, y compris celle de Peirce, sur laquelle il va pourtant s'appuyer. Il s'attelle donc à décrire le rapport image / signe, en définissant les images à partir des signes qui la composent. L'image n'est pas le signe constitué des choses ${ }^{6}$, c'est parce que les choses signifient en elle, comme "choses perçues" sous tel ou tel aspect, ou "choses percevantes" dans telle ou telle situation, à telle ou telle occasion (et parce qu'au cinéma on peut passer de l'une à l'autre sans solution de continuité ${ }^{7}$, qu'il y a image, image-action, image-affection, image-cristal, etc. $\mathrm{Si}$ bien que les composés de l'image cinématographiques:

[...] constituent une "matière signalétique" qui comporte des traits de modulation de toutes sortes, sensoriels (visuels et sonores), kinésiques, intensifs, affectifs, rythmiques, tonals, et même verbaux (oraux et écrits). 
[...] C'est une masse plastique, une matière a-signifiante et a-syntaxique, une matière non linguistiquement formée, bien qu'elle ne soit pas amorphe et soit formée sémiotiquement, esthétiquement, pragmatiquement " (1985, p. 45).

Ces trois derniers adverbes nous font songer par ailleurs à trois grandes avenues de pénétration dans le dédale des analyses deleuziennes.

Pour "sémiotiquement", on pourra entendre la première entreprise de Deleuze, qui consiste à faire la classification des signes que le cinéma a créés pour son compte.

Pour "esthétiquement ", on pourra se référer à cette deuxième entreprise qui croise la première. Entreprise qui consiste à dégager des régimes de signes, comme ces deux régimes distincts dit organique et cristallin qui produisent deux types de récit, deux types de narration, la narration et le récit véridiques, la narration et le récit falsifiants. La "matière signalétique" se travaille alors comme la glaise du sculpteur, la couleur du peintre, la langue du poète. Elle produit de grands styles d'auteur cinéaste.

Pour "pragmatiquement" enfin, on ira du côté de la genèse des signes et de leurs effets de sens "en situation". Comment les choses font signe, deviennent signes dans une situation donnée et actualisée par le film, dans des agencements, des relations qui rendent possible leur signifiance. L'analyse du mouvement, tel que le rendent et le recréent les images cinématographiques, permet de distinguer les formes des "agencements " pratiqués et inventés par les cinéastes.

C'est ainsi que Deleuze pourra comprendre le récit et la narration de la manière suivante, et comme à contre-pied des analyses narratologiques venues de la linguistique (et d'une critique littéraire fortement inspirée par elle):

Pour Metz, la narration renvoie à un ou plusieurs codes comme à des déterminations langagières sous-jacentes d'où elle découle dans l'image au titre de donnée apparente. Il nous semble au contraire que la narration n’est qu'une conséquence des images apparentes ellesmêmes et de leur combinaison directe, jamais une donnée. La narration dite classique découle directe- 
ment de la composition organique des images-mouvements (montage), ou de leur spécification en imageperception, image-affection, image-action, suivant les lois d'un schème sensori-moteur. Nous verrons que «les" formes modernes de narration découlent des compositions et des types des images-temps: même la lisibilité (1985, p. 40).

C'est encore sur l'analyse du mouvement que repose la distinction des deux régimes. Si bien que, si Deleuze cherche à définir une logique, celle-ci ne doit rien aux structures langagières. Il n'a de cesse que d'insister sur la nécessité d'abandonner le parti pris de l'analyse discursive de type structuraliste ${ }^{8}$. Les formes rencontrées de récit ne sont pas des variantes, rendues possibles par une structure discursive invariante, de type narratif, qui fonderait l'image cinématographique. En deux mots (ou un peu plus...), l'image cinématographique ne raconte ni parce qu'elle est un "énoncé " (tout au plus est-elle un "énonçable" ", aime à préciser Gilles Deleuze) ni parce qu'elle s'organise selon un code prédéterminant. Récit et narration découlent de la sorte d'images et de la sorte de montage qui combine ces images, et dépendent de la sorte du mouvement ainsi produit. Or, l'analyse du mouvement est très riche en concepts philosophiques. Sans en faire l'inventaire, soulignons simplement ici que cette analyse et cette tradition philosophique (qui ne se résume pas à la seule contribution de Bergson) seront l'assise conceptuelle de Gilles Deleuze, après qu'il aura fait table rase des outils de la linguistique.

Gilles Deleuze ne part pas non plus d'une différenciation préalablement posée entre cinéma narratif et cinéma non narratif pour décrire la narration et le récit cinématographiques. Il part du fait que l'image cinématographique présente du mouvement et du temps, soit ces signes sensori-moteurs, ou, à l'inverse, ces «opsignes" et "chronosignes» par lesquels un récit est toujours possible (et jusque dans ses occurrences les plus paradoxales, quand l'histoire s'abolit pour laisser place nette au pur devenir). Ce qui, soit dit en passant, lui permettra de ne pas faire reposer une analyse du narratif sur une distinction qui la présuppose, et de pouvoir qui plus est parler du type de narration qui prévaut 
dans le cinéma documentaire comme dans le cinéma dit de fiction, dans le cinéma expérimental comme dans le cinéma d'auteur.

Il n'y a pas de narration (ni de description) qui soit une "donnée" des images. La diversité des narrations ne peut pas s'expliquer par les avatars du signifiant, par les états d'une structure langagière supposée sous-jacente aux images en général. Elle renvoie seulement à des formes sensibles d'images et à des signes sensitifs correspondants qui ne présupposent aucune narration, mais d'où découle telle narration plutôt qu'une autre. Les types sensibles ne se laissent pas remplacer par des processus de langage. C'est en ce sens que la narration falsifiante dépend directement de l'image-temps, des opsignes et des chronosignes, tandis que la narration traditionnelle renvoie aux formes de l'image-mouvement et à des signes sensori-moteurs (1985, p. 179).

À partir des textes d'où sont extraites ces citations, il est dès lors possible de construire le tableau suivant:

\begin{tabular}{|c|c|c|c|}
\hline Recit et mpration & Formes sensibles & d'images & \\
\hline $\begin{array}{l}\text { Signes sensitifs } \\
\text { Narration } \\
\text { traditionnelle }\end{array}$ & $\begin{array}{l}\text { Formes de l'image- } \\
\text { mouvement, à savoir } \\
\text { l'image-perception, } \\
\text { l'image-action, l'image- } \\
\text { affection (pour les } \\
\text { principales) }\end{array}$ & $\begin{array}{l}\text { Signes sensori-moteurs } \\
\text { - qui font enchaîner les } \\
\text { perceptions et les } \\
\text { actions, les perceptions } \\
\text { et les expressions } \\
\text { - qui organisent } \\
\text { l'espace de l'action } \\
\text { possible: par exemple, } \\
\text { raccord de mouvement, } \\
\text { raccord de regard }\end{array}$ & $\begin{array}{l}\text { Regima } \\
\text { orgaraique }\end{array}$ \\
\hline $\begin{array}{l}\text { Narration } \\
\text { falsifiante }\end{array}$ & $\begin{array}{l}\text { Forme de l'image-temps, } \\
\text { à savoir ici l'image-cristal }\end{array}$ & $\begin{array}{l}\text { Sonsignes et opsignes } \\
\text { - ils perturbent les } \\
\text { cnchaînements, font en } \\
\text { sorte que les perceptions } \\
\text { ne se prolongent plus en } \\
\text { action (conéma de } \\
\text { voyant) } \\
\text { Chronosignes } \\
\text { - ils rendent } \\
\text { indiscernables le réel et } \\
\text { l'imaginaire, le vrai du } \\
\text { faux, l'objectif du } \\
\text { subjectif }\end{array}$ & $\begin{array}{l}\text { Régime } \\
\text { cristallin }\end{array}$ \\
\hline
\end{tabular}


Quelques précisions sur les nouveaux concepts utilisés par Gilles Deleuze s'imposent ici dans l'optique d'une présentation générale. Pour ce qui est de "la narration véridique» en premier lieu, on pourrait la décrire comme suit: premièrement, il s'agit d'une "composition organique" (montage), c'est-à-dire une composition qui procède à une "totalisation", qui agence ses parties de telle sorte qu'elles forment un tout organique; ce qui signifie que les parties sont interdépendantes, et que chacune reflète l'état de l'ensemble, et qu'à chaque moment on puisse en tirer une vision du tout. Ici, Deleuze dépasse l'idée de simple enchaînement et de linéarité qui prévaut dans la grande syntagmatique de Metz, et parle de "totalité ouverte». Deuxièmement, ce qui prévaut dans la narration véridique, ce sont des liens sensori-moteurs: ils organisent l'espace de l'action, en rapportant un personnage à un "milieu" (famille, société, cercle d'amis, pègre, culture), en dressant des obstacles à contourner, en proposant des buts et des moyens d'action. Le lien sensori-moteur, au-delà du simple raccord de mouvement, crée en définitive un certain type de monde où l'action (réaction à une situation, changement de situation) est possible. Troisièmement, et cinématographiquement parlant, la narration véridique se traduira par l'enchaînement des "imagesperceprion", des "images-action" et des «images-affection" qui constitueront à l'écran une diégèse propre au récit et à la narration véridiques (Deleuze parlera d'espace "hodologique", en référence aux études de Simondon). Véridiques, parce qu'à chaque perception, le spectateur est capable de décrire la situation, et de prévoir l'action ou de faire le lien avec elle. Pour ce faire, le cinéaste s'est donné des "images-mouvement", où le mouvement qui prime est toujours mesuré et orienté, qu'il soit mouvement de translation ou d'expression, mouvements des choses ou mouvements entre les plans. Ces différents types de mouvements donnèrent d'ailleurs plusieurs écoles de montage (le montage dialectique des Russes, le montage impressionniste des Français, le montage expressionniste des Allemands, le montage parallèle des Américains). Au niveau de la conduite du récit enfin, ce sont les enchaînements logiques et les rapports de causalité qui prévalent.

Pour ce qui est de "la narration falsifiante", elle résulte, premièrement, d'une "composition cristalline" des images. Dans cette 
composition cristalline, les images se greffent les unes aux autres, se dédoublent, forment des circuits d'échange. Il n'y a plus aucune totalisation possible. La situation qui prévaut alors est celle qui voit naître une nouvelle entité personnage/spectateur, où le personnage "impuissant" devient spectateur du monde, voyant, et où le spectateur devient personnage, c'est-à-dire connaît la situation de voyance et de suspension d'action que le personnage connaît, au cour même du rapport au monde le plus banal et le plus quotidien. Deuxièmement, la narration falsifiante ne repose plus sur des liens sensori-moteurs, ce qui signifie une dislocation du monde de l'action: de son espace et de sa temporalité propres (Deleuze parlera alors d'espace "riemanien"). À la place, surgissent des descriptions pures ${ }^{10}$, qui valent pour leur objet. Des opsignes et des sonsignes ne font plus le partage entre le réel et l'irréel, le subjectif et l'objectif". Troisièmement et cinématographiquement parlant, la narration falsifiante ne procède plus par enchaînement d'image-perception, d'image-action et d'imageaffection, mais par des circuits d'échange dans l'image-cristal, qui créent une diégèse singulière où l'action est suspendue, inhibée, où la perception se prolonge en "image-souvenir", "image-pensée " (qu'elles soient actualisées ou non à l'écran). Pour ce faire, le

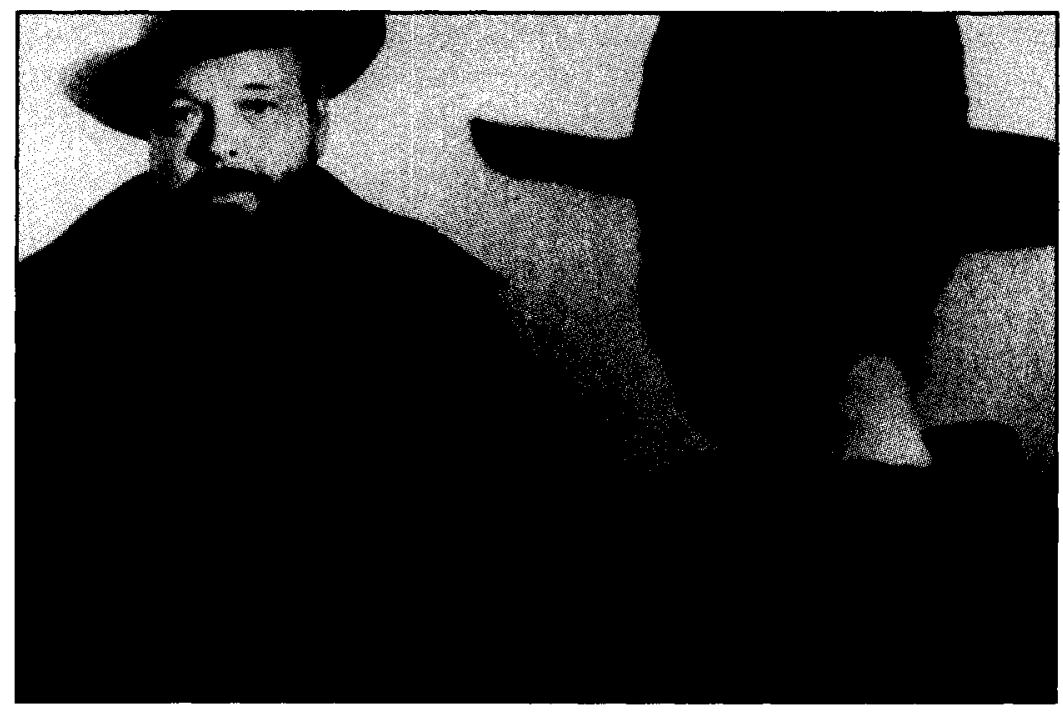

F for Fake (Orson Welles, 1973) 
cinéaste se donne des "images-temps", où le mouvement n'est plus ni mesuré ni orienté, où le mouvement est aberrant, interdisant toute connexion ou raccord d'espace, et... toute suite dans les idées, toute pensée, pulsion, sentiment qui se traduisent en action, alors qu'entre les plans, des coupures irrationnelles brisent les inférences logiques et les rapports de causalité.

Cette opposition nette et tranchée entre ces deux régimes narratifs est une première invitation à ne pas considérer la narrativité comme monolithique. Puis elle nous demande de renoncer à la définition sommaire du récit comme enchaînement événementiel produit à partir de l'enchaînement des images elles-mêmes. Il existe au moins un régime de signes où ce n'est pas l'enchaînement comme tel qui prévaut, mais l'échange, le circuit entre les images, et dans le plan, entre les images actuelles et les images virtuelles. C'est le régime de l'imagecristal, où le réel et l'imaginaire, le vrai et le faux, le subjectif et l'objectif s'échangent dans le même plan et dans le montage. Pour nous résumer, nous avons d'un côté les images-mouvement et leur enchaînement, qui définissent le cinéma classique, de l'autre côté les images-temps et le principe de l' "échange", du circuit qui définissent le cinéma moderne ${ }^{12}$.

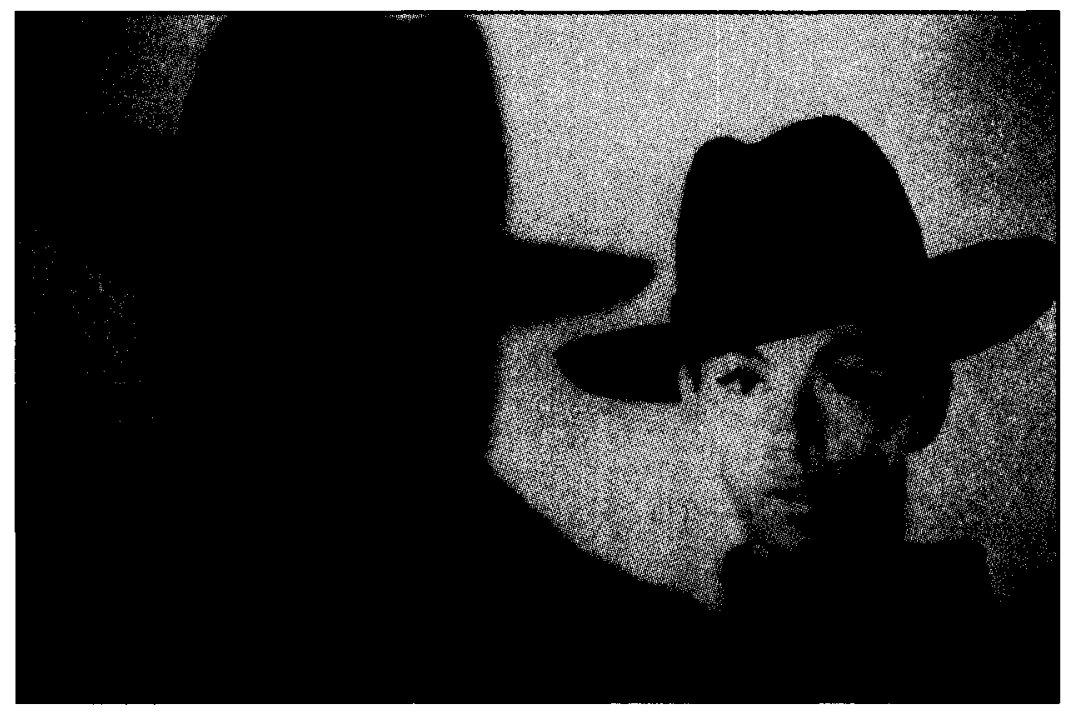

F for Fake (Orson Welles, 1973)

Deleuze et la question de la narration 141 
Il a beaucoup été question jusqu'à présent de cetre fameuse image-cristal, ou structure cristalline de l'image-temps du cinéma. Sans prétendre déplier complètement ce concept et l'explorer en détails, voyons un peu comment l'idée d'une image-cristal permet d'introduire la notion "d'indiscernabilité entre le vrai et le faux" que l'on trouve au coeur de l'analyse de la narration falsifiante et qui fait toute l'originalité de la position deleuzienne.

L'image-cristal résulte d'un processus de "cristallisation" propre au cinéma. Deleuze parle donc de structure cristalline pour décrire une image à plusieurs faces, réparties entre des faces limpides et des faces opaques, qui apparaissent en vertu de circuits entre l'image objective, présente à l'écran, ou image actuelle, et les images virtuelles qu'elle traîne avec elle, qu'elle implique ${ }^{13}$ ("images-souvenir ", "images-rêve ", "imagesmonde"). Ces images virtuelles n'ont pas besoin d'être ou d'avoir été "objectivement" présentes à l'écran à un moment donné du récit: c'est moins l'enchaînement des images qui compte ici que leur dédoublement, et, par ce dédoublement, les circuits entre images qui sont ainsi rendus possibles (en d'autres mots, l'image cinématographique présente toujours plus que son contenu propre et visible à l'écran, justement parce qu'elle entre dans des circuits d'images).

Du côté de la perception, l'image-cristal est une image qui passe constamment de son image actuelle à ses images virtuelles; ses faces limpides et ses faces opaques s'échangent au moindre changement dans son aperception. Si bien que du côté du spectateur, ces circuits entre images se resserrent à un tel point qu'il peut ne plus parvenir à discerner l'actualité de la virtualité, l'objectif du subjectif, le réel de l'imaginaire. Il ne parvient plus à statuer sur ce qu'il voit. L'objectif et le subjectif, le réel et l'imaginaire, le présent et le passé se confondent dans la même image (c’est du moins le résultat du plus petit circuit possible). Les images-temps sont par exemple des images-cristal qui produisent de la sorte des phénomènes temporels particuliers comme celui de la co-présence du passé et du futur dans le même plan, ou la co-présence de l'imaginaire, du fantasme, d'une autre image avec l'image actuelle 
(présente objectivement à l'écran) et donc la coexistence de plusieurs espaces-temps.

Avec l'image-cristal, il y a irruption de l'irréel dans la trame du réel, dans la surface du réel, comme un reflet changeant qui fait constamment passer le réel par un instant d'irréalité. Le monde visible est, pour exploiter la métaphore, comme la surface d'une eau qu'une brise légère fait miroiter, d'un reflet changeant à l'autre sous le moindre rayon de lumière. Si bien qu'au cinéma, on apprend à voir des points de vue qui coexistent et des circuits d'images, plutôt qu'à considérer les choses, les objets, et à regarder l'image comme leur tenant-lieu ou leur reproduction. C'est la croyance en l'" objectivité" des images cinématographiques (du fait de leur caractère photographique) qui n'est plus considérée comme un postulat nécessaire à la compréhension du spectateur. De même, le postulat de la lisibilité du récit à travers les seuls "enchaînements objectifs » des images est bousculé par ce concept de circuit et d'échange, qui introduit dans l'analyse, le vaste champ de la virtualité.

Le renversement est plus patent encore avec cette idée deleuzienne que l'efficacité de la narration falsifiante relève d'une "puissance du faux " qui serait propre au cinéma. Il s'agit là d'un pouvoir de remise en question de "la forme du vrai", pouvoir résultant de l'image-cristal et de la composition cristalline. Le circuit rend en effet les choses indiscernables, inassignables. Il n'y a plus de possibilité d'identification, de vérification. Nous parlions plus haut d'une nouvelle entité personnage/spectateur. Cette nouvelle entité se complique à la façon du cristal, elle acquiert une autre face où le personnage du faussaire devient le cinéma et où le cinéma acquiert la puissance du faussaire ${ }^{14}$.

Expliquer un concept deleuzien c'est, comme Deleuze le dit si bien à propos de la philosophie de Leibniz, le "déplier", se préparer à la rencontre de toutes les notions qu'il enveloppe et qui lui donnent sa consistance. Ainsi la narration falsifiante enveloppe celle de puissance du faux qui met le récit et la narration en rapport avec une forme du vrai qu'il nous reste ici à aborder, tout en restant conscients que l'analyse complète des résonances et implications philosophiques d'une telle notion feront largement défaut ${ }^{\text {is }}$ (cela nécessiterait tout un chapitre!). 
Narration véridique et récit véridique ne renvoient pas, comme on vient de le voir, au contenu du récit - on ne parle pas d'histoire vraie. Si la narration falsifiante, en tant que puissance du faux, conteste la forme du vrai, cette contestation peut prendre plusieurs formes et enclencher plusieurs récits. Premièrement, si l'on considère "les conditions de réalité d'un récit ", la forme du vrai est l'ensemble des conditions, des facteurs qui permettent d'actualiser un événement (le monde vrai est un monde tangible, préhensible, au présent, etc., où des possibles sont passés sans équivoque et sans retour de l'état virtuel à l'état actuel) : dans ce monde, l' "actualité" relève du principe de "causalité" (les choses s'actualisant dans des séries causales qui peuvent mêler cause physique et cause morale, et sur un plan chronologique qui ne permet aucun retour en arrière). L'action, telle qu'on l'entend au cinéma, suppose cette compréhension, cette organisation du monde, que l'on nomme aussi réalité, et dans laquelle on puise les critères du vraisemblable. Le monde de l'action, avec ses personnages qui agissent sur des situations et dans des situations qui changent ou qui les font changer, dont les pensées, les sentiments, s'actualisent, se prolongent en action (qu'il y ait échec ou réussite), reconduit donc cette forme du vrai sur laquelle repose le récit classique. Mais quand on ne croit plus en l'action, quand on est démuni face au monde ou que l'action ne peut plus constituer une réponse, un autre récit surgit qui repose moins sur les enchaînements que sur les plans-séquences, les temps morts, et qui relâche les liens constituant la forme du vrai des récits classiques, qui les tord, les distend. Deuxièmement, contester la forme du vrai, c'est miner l'image dite objective ou le fonctionnement objectif de l'image. À la reproduction de l'objectivité succède un approfondissement du réel, qui passe par la fabrication de récit sans assignation, sans partage possible entre le réel, l'imaginaire, le subjectif, l'objectif. Troisièmement, la narration falsifiante, parce qu'elle conteste cette forme du vrai, introduit la pensée dans l'image, en laissant l'esprit du spectateur dans l'inconfort de la non-distinction entre le vrai et le faux: les vérités d'ordre moral, logique, poétique, sociologique, politique ne s'imposent plus pour structurer le monde perçu et représenté par le cinéma. 
Quatrièmement, enfin, au niveau de la progression du récit, la narration falsifiante procédera par rupture des enchaînements logiques et des liens causals.

Est vrai ce qu'on arrive à distinguer, à discerner. Le faux, en revanche, est une puissance. C'est la puissance de rendre les choses indécidables. Une narration falsifiante n'est pas le récit d'un mensonge, c'est l'acte de fausser les paramètres, les critères du vrai, la véracité du récit. Deleuze incite donc le narratologue à réfléchir sur les notions de réel et d'irréel, de vrai et de faux. Le réel est "un ensemble de connexions légales ", "d'enchaînement prolongé des actuels» (opposé au circuit des virtuels). L'irréel est de l'ordre de "l'apparition brusque et discontinue à la conscience", "c'est un virtuel en tant qu'il s'actualise " (1990, p. 93). Le faux advient quand la distinction du réel et de l'irréel n'est plus possible. Le faux est la puissance qui rend les deux indiscernables. La puissance du faux foncrionne dans des narrations falsifiantes et des récits de simulation qui renversent le modèle du vrai reproduit par le cinéma classique et le reportage (et ceux-ci paraissent vrais "parce qu"ils reproduisent ce modèle). Du côté de la puissance du faux, et capables donc de récit et de narration falsifiants, nous aurons Welles ( $F$ for Fake), le premier des grands cinéastes falsificateurs, le "cinéma vérité " de Jean Rouch, le cinéma vécu de Pierre Perrault, ainsi que le cinéma de Shirley Clarke et celui de Cassavetes.

Pour nous résumer, et faire le point sur les deux régimes de l'image, voici le tableau suivant, confectionné à partir du chapitre VI de L'Image-temps:

\begin{tabular}{l} 
RÉGIME ORGANIQUE \\
ou régime cinétique \\
\hline la description \\
- le milieu décrit esr posé comme in- \\
dépendant de la description que la ca- \\
méra en fait et vaut pour une réalité \\
préexistante \\
- situation sensori-motrice \\
- cinéma d'actant
\end{tabular}

\begin{tabular}{l}
\hline RÉGIME CRISTALLIN \\
ou régime chronique \\
\hline la description \\
- la descriprion vaut pour son objet, le \\
remplace, le crée et le gomme à la fois, \\
et ne cesse de faire place à d'autres des- \\
criptions qui contredisent, déplacent ou \\
modifient les précédentes \\
- situation optique et sonore pure \\
- cinéma de voyant \\
\hline
\end{tabular}

Deleuze er la question de la narration 
le réel et l'imaginaire

- le réel supposé se reconnaît à sa continuité, même interrompue, aux raccords qui la rétablissent, aux lois qui déterminent les successions, les simultanéités, les permanences: c'est un régime de relations localisables, d'enchaînements actuels, de connexions légales, causales et logiques

- l'irréel, le souvenir, le rêve, l'imaginaire, sont définis par opposition: pure apparition à la conscience, actualisation dans la conscience

la narration

- développement des schèmes sensorimoteurs: des personnages réagissent à des situations, ou bien agissent de manière à dévoiler la situation

- agir : l'action suit la vision ou inversement

— narration véridique: qui prétend au vrai

- raccord de mouvement

- espace hodologique (champ de forces, d'obstacles, etc.) et euclidien

- représentation indirecte du temps qui découle de l'action, dépend du mouvement, et conclut de l'espace le réel er l'imaginaire

- l'actuel est coupé de ses enchaînements moreurs, ou le réel de ses connexions légales, et le virtuel, de son côté, se dégage de ses actualisations, se met à valoir pour lui-méme

- le réel et l'imaginaire, l'actuel et le virtuel, courent l'un derrière l'autre, échangent leur rôle et deviennent indiscernables

[image-cristal $=$ coalescence d'une image actuelle et de son image virtuelle; indiscernabilité des deux images distinctes]

la narration

- les personnages, devenus voyants, ne peuvent plus ou ne veulent plus réagir à des situations optiques et sonores pures; il faut qu'ils arrivent à voir ce qu'il y a dans la situation

- voir : la vision tient lieu d'action

- narration falsifiante: puissance du faux qui pose la simultanéité de présents incompossibles ou la coexistence de passés non nécessairement vrais; elle pose au présent des différences inexplicables; au passé des alternatives indécidables entre le vrai er le faux; le personnage du faussaire devient le personnage même du cinéma

- faux raccord de mouvement

- espace riemanien, quantique, probabilitaire, cristallisé

- relations non localisables $=$ représentation directe du temps; le temps chronique, non chronologique produit des mouvements nécessairement anormaux 
le récit véridiqute

- il procède de la discincrion entre les images subjectives (ce que voir un personnage) et les images objectives (ce que la caméra voit, c'est-à-dire, entre autres, le personnage) ; ces images finissen par s'identifier pour constituer le Vrai : on sait qui est vu et qui voit quoi, et que ce que voit la caméra correspond à ce que les personnages voient le récit de simulation

- la distinction entre les images objecrives et les images subjecrives tend à s'évanouir; on ne saic plus qui a vu quoi (la caméra, le personnage?); le personnage n'est plus réel ou fictif (voir le cinéma vérité), il fabule lui-même, il est d'autant plus réel qu'il s'invente un personnage; il y a un devenir autre du cinćaste

Ce qui prime, donc, ce sont les régimes de signes et leur puissance : les notions de récit et de narration passent finalement à la trappe. Deleuze reprend ces notions narratologiques pour mieux les évacuer, pour privilégier le concept de régime de signes et pour ouvrir tout un nouveau champ de prospectives.

N'y a-t-il même pas d'autres régimes que les deux considérés ici, le cristallin er l'organique? Évidemment, il y en a d'autres (quel est le régime des images électroniques digitales, un régime-silicium au lieu d'un régime-carbone? Là encore les arts, la science, la philosophie opéreraient des rencontres (1990, p. 95).

Pour finir ce petit tour d'horizon des principales percées narrarologiques de la philosophie deleuzienne, il serair bon de revenir sur certaines notions dont il partage l'usufruit avec les narratologues. Nous avons donc choisi ici d'étudier plus particulièrement "le point de vue " " et le couple oppositionnel "histoire et devenir".

Pour résumer succinctement la question du point de vue, nous dirons que cette notion, qui a passionné plus d'un narratologue, recouvre les principales déterminations suivantes: le point de vue déterminant un angle de vue (c'est-à-dire un emplacement de la caméra); le point de vue déterminant le statut de l'image (objectif/subjectif), le point de vue entendu comme focalisation (vision et savoir sur l'univers diégétique).

Toutes ces déterminations ont en commun d'opposer le point de vue à l'objer vu, le foyer de perception au perçu. Le travail de Gilles Deleuze consiste au contraire à penser ensemble le point de vue et l'objet. Dans cette optique, la question de l'emplacement de la caméra n'est pas déterminante pour lui (l'emplace- 
ment physique de la caméra s'évanouissant par les mouvements de caméra des plus simples aux plus compliqués, mais aussi par le montage, et le mouvement dans les plans qui prennent le dessus, en réorganisant l'espace de l'image en fonction d'eux, sans compter tous les points de vue virtuels que l'image comporte, quelle que soit leur actualisation ${ }^{17}$ ). Pour ce qui est de la détermination du statut de l'image, là encore Deleuze préfere insister, pour ce qui concerne le régime cristallin, sur la labilité du point de vue, son instabilité : les images passant du pôle objectif au pôle subjectif de façon incessante.

On peut dire que le film commence avec la distinction des deux sortes d'images [objectives et subjectives] et finit avec leur identification, leur identité reconnue. Les variations sont infinies, parce que la distinction autant que l'identité synthétique peuvent s'établir de toute sorte de façons. Il n'y en a pas moins ici les conditions de base du cinéma, du point de vue de la véracité de tout récit possible. C'est la distinction de l'objectif et du subjectif, mais aussi bien leur identification, qui se trouvent mises en question dans un autre mode de récit. [...] Dans le cinéma de poésie [tel que défini par Pasolini] la distinction s'évanouissait entre ce que voyait subjectivement le personnage et ce que voyait objectivement la caméra, non pas au profit de l'un ou de l'autre, mais parce que la caméra prenait une présence subjective, acquérait une vision intérieure, qui entrait dans un rapport de simulation ("mimesis") avec la manière de voir du personnage. [...] Les images objectives et subjectives perdent leur distinction, mais aussi bien leur identification, au profit d'un nouveau circuit où elles se remplacent en bloc, ou bien se contaminent, ou bien se décomposent et se recomposent (1985, p. 193-194).

Deleuze n'aborde enfin jamais les problèmes de focalisation indépendamment de la nature des images qui sont en jeu (de leur "régime"). Dans le régime cristallin ce qui importe ce n'est pas le point de vue sur l'objet (les information disponibles, les informations manquantes). Il n'y a pas de "focalisation" à proprement parler, il y a des descriptions qui valent pour leur objet, le remplacent, le créent et le gomment à la fois, se contre- 
disent, se modifient mutuellement. Les points de vue, en ce sens, signifient pour Deleuze des "centres de perception". Mais s'il y a à l'image une multitude de points de vue, cela ne veut pas dire qu'il y a "variation de points de vue extérieurs sur un objet supposé invariable (l'idéal du vrai serait conservé)». Le point de vue est à l'intérieur de l'objet et signifie ses métamorphoses. Ce sont les métamorphoses de l'objet qui génèrent les points de vue. Le point de vue ne définit pas l'image de l'extérieur (par rapport à une entité qui voit, sait, interprète, etc.), il est à l'intérieur de l'image, il est intérieur à la chose même. Si bien qu'on ne peut parler d'une perception vraie (donnée par le bon point de vue) opposée aux apparences (qui sont des faux points de vue sur les choses) dans les récits de simulation et les narrations falsifiantes. Le point de vue est la projection sur un plan d'une métamorphose de l'objet, "[...] l'objet lui-même n'étant à la limite que la connexion de ses propres projections, la collection ou la série de ses propres métamorphoses" (1985, p. 187).

Quel est, en deuxième lieu, le sens de l'opposition deleuzienne entre "histoire» et "devenir " ${ }^{18}$ ? Dans le régime cristallin, nous dit Deleuze, nous avons plus affaire à du devenir qu'à de l'histoire. On ne saurait développer ici l'extraordinaire fortune de ce concept dans la pensée deleuzienne (voir le chapitre 10 "Devenir-intense, devenir-animal, devenir-imperceptible" de Mille Plateaux $\left.{ }^{19}\right)$. Restreignons-nous donc à une seule question: comment l'opposition histoire / devenir fait-elle sens au cinéma?

Il faut distinguer plusieurs niveaux: au premier niveau, l'histoire, fondée sur la possibilité de l'action (il y a des conditions de changement, des causes de changement, des changements, des conséquences de ces changements) est opposée au devenir, où les changements sont décrits pour eux-mêmes comme manifestations directes du temps. À un deuxième niveau, au lieu de fixer, circonscrire les images pour qu'elles racontent des histoires, il s'agit de faire aussi "devenir" les images (les faire passer d'un pôle à l'autre, de l'image actuelle à l'image virtuelle, etc.) en ôtant au mouvement ces centres de force, d'équilibre, etc. 
Il y a chez Welles une mutation cinématographique non moins que métaphysique. Car ce qui s'oppose à l'idéal de vérité, ce n'est pas le mouvement: le mouvement reste parfaitement conforme au vrai tant qu'il présente des invariants, point de gravité du mobile, points privilégiés par lesquels il passe, point de fixité par rapport auquel il se meut. C'est pourquoi l'image-mouvement, dans son essence même, est justiciable de l'effet de vérité qu'elle invoque tant que le mouvement conserve ses centres. Et c'est ce que nous essayons de dire depuis le début de cette étude: une mutation cinématographique se produit lorsque les aberrations de mouvement prennent leur indépendance, c'est-à-dire lorsque les mobiles er les mouvements perdent leurs invariants. Alors se produit un renversement où le mouvement cesse de se réclamer du vrai et où le temps cesse de se subordonner au mouvement: les deux a la fois. Le mouvement fondamentalement décentré devient fiux mouvement, et le temps fondamentalement libéré devient puissance du faux qui s'effectue maintenant dans le faux mouvement (Arkadin toujours déja là). Welles semble le premier a avoir ouvert cette brèche, où allaient s'introduire le néoréalisme et la Nouvelle Vague, avec des moyens tout différents (1985, p. 186-187).

Le troisième niveau, enfin, est celui du récit dit "de simulation". Là encore il faut se souvenir de l'équation suivante: est vrai ce qui est distingué, identifié, poser dans sa permanence. Le récit de simulation opère le "devenir autre». Il ne s'agit pas de raconter une expérience, des événements avec plus ou moins de discours, d'art, de couches de représentation (on se souvient de la grande affaire du réalisme, la transparence, faire en sorte que les événements semblent se raconter d'eux-mêmes). Le «devenir autre" c'est l'expérience du "Je est un autre". Expérience d'une limite, d'une métamorphose. Le "devenir autre" affecte les personnages, le spectateur, le cinéaste, à des degrés et selon des modalités divers, mais ne se résout ni dans l'imitation ni dans l'identification (deux termes étant identifiés, l'un s'identifie à l'autre par projection, il ne passe alors dans l'autre qu'en imagination); son principe n'est pas la ressemblance, la répétition ou la reproduction, mais la simulation, la fabulation. La part de 
création est dès lors prépondérante dans ce procès où je ne deviens autre qu'en participant activement à cette transformation.

Le devenir ne produit pas autre chose que lui-même, c'est une fausse alternative qui nous fait dire: ou bien l'on imite, ou bien l'on est. Ce qui est réel, c'est le devenir luii-même, le bloc de devenir, et non pas des termes supposés fixes dans lesquels passerait celui qui devient $(1980$, p. 291).

Lidentification du spectateur, par exemple, est constamment déstabilisée par le devenir autre des personnages et du cinéaste, par le jeu des métamorphoses. Pour illustrer ce point, prenons deux situations opposées de confrontation à l'autre, développées au cinéma. Dans le documentaire ethnographique classique, chacun est ciblé, identifié: le cinéaste anthropologue drapé de son objectivité scientifique, la population visée, sommée de décliner son identité à travers ses pratiques et ses coutumes, les spectateurs venus reconnaître soit que ces populations ont décidément des mours "étranges", soit que leur mode de fonctionnement et leur culture présentent d'"étranges" similitudes avec les leurs. C'est un cas de polarisation de l'identification en fonction de trois identités bien définies. L'identification à l'autre qui pourrait être à l'œuvre ici, qu'elle touchât le cinéaste, les spectateurs, ou même les personnes filmées, n'est en aucun cas assimilable à ce "devenir autre" dont parle Deleuze, puisque le discours filmique ethnographique classique se fonde tout au contraire sur l'intangibilité de la distinction des trois parties prenantes de cette expérience documentaire. Ici, nulle question de changement d'identité. Deleuze va donc opposer aux démarches dites scientifiques du reportage ethnographique un tout autre cas de figure, caractéristique selon lui des films de Rouch et de Perrault:

[Rouch et Perrault] doivent devenir autres, avec leurs personnages, en même temps que leurs personnages doivent devenir autres eux-mêmes. La formule célèbre: "ce qui est commode avec le documentaire, c'est qu'on sait qui on est et qui on filme" cesse d'être valable. La forme d'identité Moi = Moi (ou sa forme dégénérée,

Deleuze et la question de la narration 
eux $=$ eux) cesse de valoir pour les personnages et les cinéastes, dans le réel aussi bien que dans la fiction. Ce qui se laisse deviner plutôt à des degrés profonds, c'est le "Je est un autre" de Rimbaud. Godard le disait à propos de Rouch: non seulement pour les personnages eux-mêmes, mais pour le cinéaste qui, "blanc tout comme Rimbaud déclare lui aussi que “je est un autre", c'est-à-dire Moi, un Noir» (1985, p. 199).

Pour conclure, nous soulignerons d'abord que la ligne de séparation entre le cinéma classique et le cinéma moderne passe, dans L'Image-mouvement et L'Image-temps, par la crise de l'image-action. C'est dire comme le narratif est au centre des débats. Mais Deleuze ne joue pas un cinéma narratif contre un cinéma pensant. D’un côté, il est vrai que la perception doit se prolonger en pensée (et non en action). Si cela affecte l'histoire (c'est-à-dire le cours événementiel), cela ne supprime pas le récit. Deleuze différencie les récits, jusqu'au point où s'abolit l'histoire. Il le fait en dépliant toutes les potentialités de l'imagetemps, tout ce qu'elle permet en libérant l'image cinématographique des contraintes de l'action. Cela le conduit évidemment jusque dans les eaux du cinéma expérimental. Dans la perspective deleuzienne, le cinéma moderne ne fait que développer les potentialités d'un type d'image, mais ces potentialités pourront se retrouver dans un cinéma qui ne sera pas forcément à l'image de ceux de Godard, de Garel, etc., qui occupent une place de choix dans les analyses de L'Image-mouvement et de L'Image-temps. Dans la deuxième partie du deuxième tome (à partir du chapitre 7), nous touchons à un autre aspect de l'image-temps, l'image pensante. Le narratif passe à l'arrièreplan. Le récit n'est plus le truchement de la pensée, la pensée se développe directement par et dans l'image à la faveur d'un travail sur le médium lui-même, comme on travaille dans la langue, avec elle ou contre elle pour faire surgir des concepts.

Université de Montréal 


\section{NOTES}

1 "Commen s'opire le passage d'une narration orale ou écrite à une narration audiovisuelle? Comment passe-t-on de l'acte de raconter verbalement à celui de raconter en montrant? Qu'est-ce que ha visualisation d'un récit? Qui raconte le film? Quel est le starut des images et des sons dans un film narratif? s'agit-il d'une illustration objective? (Qui voir les images du film?" (André Gaudreault et François Jost, Le Récit cinématographiqute, Paris: Nathan, 1990, p.7).

2 Voir André Gitudreault et François Jost, Le Récit cinématographique (Paris: Nathan, 1990); André Gaudreault, Du littératre au filmique (Québec/Paris: Nota bene/Armand Colin, 1999); Michel Marie et Marc Vernet (direcrion), Metz et la théorie du cinénum, laris, Jris et Mérictiens Klincksieck, 1990.

3 Voir notamment ha reproduction des entretiens yu'il a accordés al l'occasion de la parution de ses deux liveres sur le cinéma dans l'ourparlers (1'uris: Minuir, 1990).

4 Metz écrit dans son célèbre essiti " le cinéma, langue ou langage?" : Le film est trop clairment un nessage pour qu'on ne lui suppose pas un coden (Essais sur la synification an cimina, tome 1. Paris: Klincksieck, 1968, p. 48). Autres citations pertinentes dans le mene chapitre, quant au rôle des concepts issus de la linguistique er de l'analyse structuale dans la compréhension de la narration filmique: a) « [... la bande elle-même raconte toujours déjà quelque chosen; b) le cinéma n'a pas de mots, "[...] l'image (du moins celle du cinćma) équivaut à une ou plusieurs phrases, et la séquence est un segranent complexe de discours"; c) il faut penser "[...] un système signitiant proprencent cinématographiquen (p. 53).

5 "L'unité réelle minima, ce r'est pas le mot ou l'idée, ni le concept, ni le signifiant, mais l'agencement. C"est toujours un agencement qui produit les énoncés" (Gilles Delenze, Diatogues, l'aris: Chramps Flammarion, 1993, p. 65).

6 En cela, (Billes Ddeuze soppesse netrement aux propositions de Christian Metz: "(i. Ryte se moque d'une cerraine conception naïve de la langue (que condamnat déja Situssure) er qu'il baprise ironiquement "The FIDO-fido theory": au chien Fido correspond rigourcusement le nom $\mathrm{FHDO}$; les mots nomment après coup, chacun à chacun, des choses en nombre égal et strictement préexistantes; cette façon de voir, très dépassée en linguistique, l'est beancoup moins au cinéma; il y a autant de "choses" dans l'image filmique qu'il y en avait dans le spectacle filmé." Er: "Un spectacle visuel entraine une adhérence du signifiant et du signifié" (Essats sur la signification ale cincina, tone 1, Paris: Klincksicek, 1968, p. 69).

7 Voir à ce propos l'urilisation delcuzienne des propositions de Bergson pour penser la perception cinématographique, dans L'Image-mouvement (Paris: Minuit, 1983).

8 Gilles Deleuze prend te parti de Pasolini plutôt que celui d'Eco, qui accusait Pasolini d’ingúnuté sćmiorique. La "matière signalériqu"”, dont parle Gilles Deleuze, est la base du "langage de la réalité (12asolini), concept impossible à entendre pour un sémioricien de la trempe d'Eco: «Domant a cer objer réel présumé la fonction de signifiant, l'asolini ne distingue pas clairement signe, signifiant, signifié et référent; et sil est une chose que la sémiotique ne peut absolument pas accepter, c'est bien la substinution du référnt au signifie" (La Structure absente, Paris: Mercure de France, 1972, p. 225). Toute l'entreprise deleuzienne consiste justement à penser le signe hors de cette structure triangulaite bien connue de la sémiotique d'inspiration saussurienne.

9 Il faudrait tout un chapitre pour développer les implications d'une telle notion: "Cé n'est pas une énonciarion, ce ne sont pas des énoncés. C'est un "énonçable". Nous voulons dire que, lorsque le langage s'empare de certe matière (et il le fait nécessaitement), alors elle donne lieu à des énoncés qui viennent dominer ou même remplacer les images et les signes, et qui renvoient pour leur compte à des traits 
pertinents de la langue, syntagmes et paradigmes, tout différents de ce d'où on était parti. Aussi devons-nous définir, non pas la sémiologie mais la "sémiotique", comme le système des images et des signes indépendamment du langage en général "(Gilles Deleuze, L'Image-temps, Paris: Minuit, 1985, p. 44).

10 C'est dans ce passage consacré au type de description de la marration falsifiante que Gilles Deleuze fait sa référence au nouveau roman. Ce qui explique aussi en partie la place importante qu'il accorde au cinéma de Robbe-Grillet, place qui en a étonné plus d'un parmi les critiques et les théoriciens.

11 "Ce n'est pas l'image-souvenir ou la reconnaissance attentive qui nous donne le juste corrélat de l'image optique-sonore, ce sont plutôt les troubles de la mémoire et les échecs de la reconnaissance. C'est pourquoi le cinéma européen s'est confronté très tôt à un ensemble de phénomènes, amnésie, hypnose, hallucination, délire, vision des mourants, et surtout cauchemar et rêve [...]. Le cinéma européen y voyait un moyen de rompre avec les limites "américaines" de l'image-action, et aussi d'atreindre à un mystère du temps, d'unir l'image, la pensée et la caméra dans une même subjectivité automatique, par opposition à la conception trop objective des Américains" (Gilles Deleuze, L'Image-temps, Paris: Minuit, 1985, p. 75-76).

12 Sans vouloir insister sur le caractère historique de ce changement de régime, rappelons l'idée deleuzienne d'une crise de l'image-action qui se scrait d'abord produite en Italie (avec le néoréalisme, 1948), puis en France (avec la Nouvelle Vague, 1958), et en Allemagne (1968). En Iralie, pour saisir la vie populaire sous-jacente à loppression, il fallait "un nouveau type de "récit", capable de comprendre l'elliptique et l'inorganisé".

13 "Le cinéma ne présente pas seulement des images, il les entoure d'un monde. C'est pourquoi il a cherché très tôt des circuits de plus en plus grands yui uniraient une image actuelle à des images-souvenir, des images-rêve, des images-monde " (Gilles Deleuze, L'Image-temps, Paris: Minuit, 1985, p. 92).

14 Notamment F for Fake de Welles (1974).

15 On peut noter en passant que l'origine de ce concept, forme du vrai, se trouve, pour ce qui concerne l'utilisation qu'en fait Deleuze, dans la philosophic nietzschéenne.

16 Pour une étude complete de la notion de point de vue, il faudrait commencer par expliquer tout ce que l'analyse de Gilles Deleuze doit au concept leibnizien du point de vue (qui n'est jamais pensé en terme de restriction mais de variation).

17 À la limite, il n'est pas besoin que la caméra adopre effectivement le point de vue de tel personnage, ou le point de vue neutre er omniscient, la seule présence dans le champ de personnages multiplie les points de vue. Il n'y a pas que l'angle de la caméra qui compte, et la focalisation n'est pas rivée à l'image dite actuelle, à l'écran, présente. Nous ne voyons pas les images sans les virtualités qu'elles impliquent comme autant de points de vues.

"Mes relations avec les choses se rrouvent elles-mêmes dénaturées par ma solitude. Lorsqu'un peintre ou un graveur introduit des personnages dans un paysage ou à proximité d'un monument, ce n'est pas par goût de l'accessoire. Les personnages donnent l'échelle et, ce qui importe d'avantage encore, ils constituent des points de vue possibles qui ajoutent au point de vue réel de l'observateur d'indispensables virtualités." (Michel Tournicr, Vendredi ou les limbes du Pacifique, Paris, Gallimard, 1996, p. 53).

18 "L'écrivain tord le langage, le fait vibrer, l'érreint, le fend, pour arracher le percept aux perceprions, l'affect aux affections, la sensation aux opinions. $|.$.$| préci-$ sément, c'est la tâche de tout art, et la peinture, la musique, n'arrachent pas moins aux couleurs et aux sons les accords nouvcaux, les paysages plastiques ou mélodiques, 
les personnages rythmiques qui les élèvent jusqu'au chant de la terre et au cri des hommes: ce qui constitue le ton, la santé, "le devenir, un bloc visuel et sonore" " (Gilles Deleuze, Qu'est-ce que la philosophie?, Paris: Minuit, 1991, p. 167). C'est moi qui souligne.

19 Deleuze ouvre d'ailleurs ce chapirre par une référence cinématographique dans un premier paragraphe inticulé "Souvenir de spectateur" où il raconte le film suivant : "Je me souviens du beau film Willard (Daniel Mann, 1972). Peut-être une série B, mais un beau film impopulaire, puisque les héros sont des rats. Mes souvenirs ne sont pas forcément exacts. Je raconte l'histoire en gros. Willard vit avec sa mère autoritaire dans la vieille maison de famille. Épouvantable atmosphère cedipienne. Sa mère lui ordonne de détruire une portée de rats. Il en épargne un ou deux (ou quelques-uns). Après une violente dispute, la mère qui "ressemble" à un chien, meurt. Willard risque de perdre la maison convoitée par un homme d'affaires. Willard aime le rat qu'il a sauvé, Ben, qui se révèle d'une prodigieuse intelligence. Il y a de plus une rate blanche, la compagne de Ben. Rentré du bureat, Willard passe tout son temps avec eux. Ils ont maintenant pullulé. Willard conduit la meute de rats sous le commandement de Ben, chez l'homme d'affaires et le fait mourir atrocement. Mais, emmenant ses deux préférés au bureau, il commet une imprudence, et doit laisser les employés tuer la blanche. Ben s'échappe, après un long regard fixe et dur sur Willard. Alors celui-ci connait une pause dans son destin, dans son devenir rat. De toutes ses forces, il tente de rester parmi les humains. Il accepte même les avances d'une jeune fille de buteau qui "ressemble" beaucoup à une rate, mais justement ne fait pas qu'y ressembler. Or un jour où il a invité la jeune fille, prêt à se faire conjugaliser, reodipianiser, il revoit Ben qui surgit, haineux. Il tente de le chasser, mais chasse en fait la jeune fille, et descend dans la cave où Ben l'attire, où l'attend une meute innombrable pour le dépecer. C'est comme un conte, ce n'est jamais angoissant" (Mille plateaux, Paris: Minuit, 1980, p. 285).

\section{OUVRAGES CITÉS}

Deleuze, Gilles. L'Image-mouvement. Cinéma 1. Paris: Minuir, 1983.

Deleuze, Gilles. L'Image-temps. Cinéma 2. Paris: Minuit, 1985.

Deleuze, Gilles. Pourparlers. Paris: Minuit, 1990.

Deleuze, Gilles et Felix Guattari. Mille phateaux. Capitalisme et schizophrénie. Paris: Minuit, 1980.

Deleuze, Gilles et Félix Guattari. Qu'est-ce que la philosophie? Paris: Minuit, 1991.

Deleuze, Gilles et Claire Parnet. Dialogues. Paris: Flammarion, 1996.

Eco, Umberto. La Structure absente. Paris: Mercure de France, 1972.

Gaudreault, André et François Jost. Le Récit cinématographique. Paris: Nathan, 1990.

Metz, Christian. Essais sur la signification an cinéma, tome 1. Paris: Klincksieck, 1968. 